\title{
The Mittag-Leffler function and its application to the ultra-hyperbolic time-fractional diffusion-wave equation
}

\section{Gustavo A. Dorrego}

To cite this article: Gustavo A. Dorrego (2016) The Mittag-Leffler function and its application to the ultra-hyperbolic time-fractional diffusion-wave equation, Integral Transforms and Special Functions, 27:5, 392-404, DOI: 10.1080/10652469.2016.1144185

To link to this article: http://dx.doi.org/10.1080/10652469.2016.1144185

曲 Published online: 07 Feb 2016.

Submit your article to this journal $\pi$

ЏII Article views: 121

Q View related articles $\square$

View Crossmark data ¿

Citing articles: 1 View citing articles $\sqsubset$ 


\title{
The Mittag-Leffler function and its application to the ultra-hyperbolic time-fractional diffusion-wave equation
}

\author{
Gustavo A. Dorrego a \\ ${ }^{a}$ Department of Mathematics, Faculty of Exacts Sciences, Northeast National University, Corrientes, Argentina
}

\begin{abstract}
In this paper we study an $n$-dimensional generalization of timefractional diffusion-wave equation, where the Laplacian operator is replaced by the ultra-hyperbolic operator and the time-fractional derivative is taken in the Hilfer sense. The analytical solution is obtained in terms of the Fox's H-function, for which the inverse Fourier transform of a Mittag-Leffler-type function that contains in its argument a positive-definite quadratic form is calculated.
\end{abstract}

\section{ARTICLE HISTORY}

Received 9 October 2015

Accepted 17 January 2016

\section{KEYWORDS}

Fractional differential equation; Hilfer fractional derivative; Caputo fractional derivative;

Riemann-Liouville fractional derivative;

Mittag-Leffler-type function; Fox's H-function; integrals transforms; ultra-hyperbolic operator

\section{AMS SUBJECT CLASSIFICATION}

26A33; 33E12; 33E20; 35R11

\section{Introduction}

The diffusion-wave equation has been studied by many authors in the fractional calculus context since it was observed that it could interpolate between heat and waves diffusion processes. Pioneering work on this type of equation may be mentioned: Fujita,[1] Nigmatullin,[2] Wyss,[3] Schnaider and Wyss [4] and Mainardi.[5] Fundamentally, according author's knowledge, the generalizations of the heat and wave equations have been studied in which the time derivative has been replaced by a fractional derivative of real order following Riemann-Liouville definitions and Caputo definition,[6,7] or the Hilfer definition, which contains as a particular case both.[8,9] Also one-dimensional generalizations have been studied in which the ordinary derivative in the space variable has been replaced by the Riesz derivative (see, e.g. [10]). The $n$-dimensional generalizations have been proposed considering fractional power of the Laplace operator on the space variable.[11] Other $n$-dimensional generalization, where the Laplacian operator was replaced by another of elliptic type, $[12,13]$ were also proposed.

On the other hand, in 2003, Nonlaopon and Kananthai [14] proposed and studied an $n$ dimensional generalization (but not in the fractional calculus context) of the heat equation in which the Laplacian operator was replaced by the ultra-hyperbolic operator. Following 
the same idea, Sansanit and Kananthai [15] proposed a generalization of the wave equation. Motivated by works mentioned in the previous paragraphs the main objective of this paper is to propose and to solve a Cauchy problem, that contains, as particular case, the equations studied in $[14,15]$. For this purpose, we replace the ordinary time derivative by a fractional derivative of order $\alpha(1<\alpha<2)$ and type $r(0 \leq r \leq 1)$ in the Hilfer sense and maintain the ultra-hyperbolic operator in the space variable. The objective of using the Hilfer derivative is to include two Cauchy problems that could arise separately and where the ordinary time derivative is replaced by Riemann-Liouville and by Caputo fractional derivatives. To solve the equation, we follow the technique developed by Yakubovich and Luchko , [16] which consists in applying Laplace and Fourier transform together with the Mellin transform. This technique has proven a powerful tool for solving fractional partial differential equations, as seen for example in [17-20].

The paper is organized as follows. Section 2 provides the definitions of differential and integral operators of non-integer order, Mittag-Leffler function, the Laplace transform, the Fourier transform and Mellin transform. In Section 3 the diffusion-wave equation is solved and inverse Fourier transform of a Mittag-Leffler-type function which contains in its argument a positive-definite quadratic form is calculated. Then several special cases are analysed.

\section{Preliminary results}

\subsection{Mittag-Leffler function}

It is known the distinguished role played by the Mittag-Leffler function in solving differential equations of non-integer order. This function is that a generalization of the exponential function was introduced by the Swedish Mathematician G. Mittag-Leffler in 1903 and is given by

$$
E_{\alpha}(z)=\sum_{n=0}^{\infty} \frac{z^{n}}{\Gamma(\alpha n+1)}, \quad \alpha, z \in \mathbb{C}, \Re(\alpha)>0 .
$$

In 1905, Wiman studied the following generalization to two parameters of (2.1) :

$$
E_{\alpha, \beta}(z)=\sum_{n=0}^{\infty} \frac{z^{n}}{\Gamma(\alpha n+\beta)}, \quad \alpha, \beta, z \in \mathbb{C}, \Re(\alpha)>0, \Re(\beta)>0
$$

The Mittag-Leffler function has been studied by many authors who have proposed and studied various generalizations and applications. A very interesting work that meets many results about this function is due to Haubold et al .[21]

\subsection{Fox's H-function.}

The Fox's H-function is another of the so-called special functions of the fractional calculus and contains as particular case the Mittag-Leffler function. The H-function was introduced by Fox [22] as generalizations of the Meijer function. Here we adopt the definition and properties mentioned in [23] with minimal modifications regarding notation. 
The H-function is defined by means of a Mellin-Barnes-type integral in the following manner :

$$
\begin{aligned}
H_{p, q}^{m, n}\left(z \mid \begin{array}{l}
\left(a_{1}, A_{1}\right), \ldots,\left(a_{p}, A_{p}\right) \\
\left(b_{1}, B_{1}\right), \ldots,\left(b_{q}, B_{q}\right)
\end{array}\right) & =H_{p, q}^{m, n}\left(z \mid \begin{array}{c}
\left(a_{p}, A_{p}\right) \\
\left(b_{q}, B_{q}\right)
\end{array}\right) \\
& =\frac{1}{2 \pi i} \int_{\mathcal{C}} \Theta(s) z^{-s} \mathrm{~d} s
\end{aligned}
$$

where the integrand is

$$
\Theta(s)=\frac{\Pi_{j=1}^{m} \Gamma\left(b_{j}+B_{j} s\right) \Pi_{k=1}^{n} \Gamma\left(1-a_{k}-A_{k} s\right)}{\Pi_{k=n+1}^{p} \Gamma\left(a_{k}+A_{k} s\right) \Pi_{j=m+1}^{q} \Gamma\left(1-b_{j}-B_{j} s\right)},
$$

where $i=(-1)^{1 / 2}, z \neq 0$, and $z^{-s}=\exp [-s\{\ln |z|+\mathrm{i} \arg z\}]$, where $\ln |z|$ represents the natural logarithm of $|z|$ and $\arg z$ is not necessarily the principal argument.

In (2.4), an empty product is always interpreted as unity; $m, n, p, q \in \mathbb{N}_{0}$, with $0 \leq n \leq p, 1 \leq m \leq q, A_{k}, B_{j} \in \mathbb{R}^{+}, a_{k}, b_{j} \in \mathbb{R}$ or $\mathbb{C}, k=1, \ldots, p ; j=1, \ldots, q$.

The contour $\mathcal{C}$ starting at the point $p-\mathrm{i} \infty$ and going to $p+\mathrm{i} \infty$ where $p \in \mathbb{R}$ such that all the poles of $\Gamma\left(b_{j}+B_{j} s\right)(j=1, \ldots, m)$, are separated from those of $\Gamma\left(1-a_{k}-A_{k} s\right)$ $(k=1, \ldots, n)$.

The integral is convergent in any of the following cases :

(1) $C>0,|\arg z|<\frac{1}{2} \pi C$ and $z \neq 0$;

(2) $C=0, p D+\mathfrak{R}(E)<-1, \arg z=0$ and $z \neq 0$

where

$$
\begin{aligned}
C & =\sum_{j=1}^{n} A_{j}-\sum_{j=n+1}^{p} A_{j}+\sum_{j=1}^{m} B_{j}-\sum_{j=m+1}^{q} B_{j} \\
D & =\sum_{j=1}^{q} B_{j}-\sum_{j=1}^{p} A_{j}
\end{aligned}
$$

and

$$
E=\sum_{j=1}^{q} b_{j}-\sum_{j=1}^{p} a_{j}+\frac{p-q}{2} .
$$

A more detailed study about the $\mathrm{H}$-function can be seen at [23]. We only mentioned some properties that will be used in this paper.

\subsubsection{Properties of $\mathrm{H}$-function.}

$$
H_{p, q}^{m, n}\left(z \mid \begin{array}{l}
\left(a_{p}, A_{p}\right) \\
\left(b_{q}, B_{q}\right)
\end{array}\right)=H_{q, p}^{n, m}\left(\begin{array}{l|l}
1 & \left(1-b_{q}, B_{q}\right) \\
z & \left(1-a_{p}, A_{p}\right)
\end{array}\right),
$$




$$
\begin{gathered}
H_{p, q}^{m, n}\left(z \mid \begin{array}{c}
\left(a_{p}, A_{p}\right) \\
\left(b_{q}, B_{q}\right)
\end{array}\right)=c H_{p, q}^{m, n}\left(z^{c} \mid \begin{array}{c}
\left(a_{p}, c A_{p}\right) \\
\left(b_{q}, c B_{q}\right)
\end{array}\right), \quad c \in \mathbb{R}^{+}, \\
z^{\rho} H_{p, q}^{m, n}\left(z \mid \begin{array}{c}
\left(a_{p}, A_{p}\right) \\
\left(b_{q}, B_{q}\right)
\end{array}\right)=H_{p, q}^{m, n}\left(z \mid \begin{array}{c}
\left(a_{p}+\rho A_{p}, A_{p}\right) \\
\left(b_{q}+\rho B_{q}, B_{q}\right)
\end{array}\right), \quad \rho \in \mathbb{C} .
\end{gathered}
$$

\subsection{Brief review of fractional calculus}

In this section, we make a brief review of definitions about fractional calculus and integral transforms to be used in this work.

Definition 2.1 (Riemann-Liouville integral): Let $f \in L_{l o c}^{1}[a, b] \quad$ where $-\infty \leq a<t<b \leq \infty$. The Riemann-Liouville integral of order $v$ is defined as

$$
I^{v} f(t):=\frac{1}{\Gamma(\nu)} \int_{a}^{t}(t-\tau)^{\nu-1} f(\tau) \mathrm{d} \tau=\left(f * j_{v}\right)(t), \quad v>0 .
$$

where $j_{v}(t)=t^{v} / \Gamma(v), t>0$.

When $v=0$, is defined

$$
I^{0} f(t)=f(t)
$$

Definition 2.2 (Riemann-Liouville derivative): Let $v \in \mathbb{R}$ such that $n-1<v \leq n$, $n \in \mathbb{N} ; f \in L^{1}[a, b]$ and $f * j_{n-v} \in W^{n, 1}[a, b] ;$ where $-\infty \leq a<t<b \leq \infty$ and

$$
W^{n, 1}[a, b]=\left\{f \in L^{1}[a, b]: \frac{\mathrm{d}^{n}}{\mathrm{~d} t^{n}} \in L^{1}[a, b]\right\} .
$$

The Riemann-Liouville derivative of order $v$ is defined by

$$
R-L D^{v} f(t)= \begin{cases}\frac{\mathrm{d}^{n}}{\mathrm{~d} t^{n}} I^{n-v} f(t), & \text { if } n-1<v<n ; \\ \frac{\mathrm{d}^{n}}{\mathrm{~d} t^{n}} f(t), & \text { if } v=n .\end{cases}
$$

Definition 2.3 (Caputo derivative): Let $v \in \mathbb{R}$ such that $n-1<v \leq n, n \in \mathbb{N}$, and $f \in A C^{n}[a, b]$. The Caputo derivative of order $v$ is given by

$$
{ }^{C} D^{v} f(t)= \begin{cases}I^{n-v} \frac{\mathrm{d}^{n}}{\mathrm{~d} t^{n}} f(t), & \text { if } n-1<v<n ; \\ \frac{\mathrm{d}^{n}}{\mathrm{~d} t^{n}} f(t), & \text { if } v=n .\end{cases}
$$

Where $A C^{n}[a, b]=\left\{f:[a, b] \rightarrow \mathbb{R}: \frac{\mathrm{d}^{n-1}}{\mathrm{~d} x^{n-1}} f(x) \in A C[a, b]\right\}$, and $A C[a, b]$ is the space of absolutely continuous functions. 
Definition 2.4 (Hilfer derivative): Let $\alpha \in \mathbb{R}$ such that $n-1<\alpha \leq n, n \in \mathbb{N}$, and $\beta \in \mathbb{R}$ such that $0 \leq \beta \leq 1 ; f \in L^{1}[a, b]$ and $f * j_{(1-\beta)(n-\alpha)} \in A C^{1}[a, b]$ where $-\infty \leq a<t<b \leq \infty$. The Hilfer derivative of order $\alpha$ and type $\beta$ of $\mathrm{f}$ is given by

$$
D^{\alpha, \beta} f(t)=\left(I^{\beta(n-\alpha)} \frac{\mathrm{d}^{n}}{\mathrm{~d} t^{n}}\left(I^{(1-\beta)(n-\alpha)} f\right)\right)(t) .
$$

Remark 1: In this paper we take $a=0$ for lower limit at the Riemann-Liouville fractional integral (2.8).

Remark 2: We note that Definition 2.4 contains as particular cases Definitions 2.2 and 2.3. Indeed:

- If $\beta=0$, then

$$
D^{\alpha, 0} f(t)=\left(\frac{\mathrm{d}^{n}}{\mathrm{~d} t^{n}}\left(I^{n-\alpha} f\right)\right)(t)={ }^{R-L} D^{\alpha} f(t)
$$

- If $\beta=1$, then

$$
D^{\alpha, 1} f(t)=\left(I^{n-\alpha} \frac{\mathrm{d}^{n}}{\mathrm{~d} t^{n}} f\right)(t)={ }^{C} D^{\alpha} f(t)
$$

- If $\alpha=n$, then

$$
D^{n, \beta} f(t)=\frac{\mathrm{d}^{n}}{\mathrm{~d} t^{n}} f(t)
$$

Definition 2.5 (Laplace transform): Let $f: \mathbb{R}^{+} \rightarrow \mathbb{R}$ an exponential order and piecewise continuous function, then the Laplace transform of $\mathrm{f}$ is

$$
\mathcal{L}\{f(t)\}(s):=\tilde{f}(s)=\int_{0}^{\infty} \mathrm{e}^{-s t} f(t) \mathrm{d} t .
$$

The integral exist for $\operatorname{Re}(s)>0$.

An important property that we use in this work is the Laplace transform of the Hilfer fractional derivative, which is given by the following lemma.

Lemma 2.6 (cf. [24], Laplace transform Hilfer's derivative): Let $n \in \mathbb{N}$ and $\alpha \in \mathbb{R}$, such that $n-1<\alpha \leq n$ and $0 \leq \beta \leq 1$; then the Laplace transform of Hilfer derivative is given by

$$
\mathcal{L}\left\{D^{\alpha, \beta} f(t)\right\}(s)=s^{\alpha} \mathcal{L}\{f(t)\}(s)-\sum_{k=0}^{n-1} s^{n-k-1-\beta(n-\alpha)} \frac{\mathrm{d}^{k}}{\mathrm{~d} t^{k}} I^{(1-\beta)(n-\alpha)} f(0) .
$$

Definition 2.7 (cf.[25], Mellin transform): Let $f \in L_{l o c}^{1}(0, \infty)$ The Mellin transform of $f(t)$ is defined by

$$
\mathcal{M}\{f(t)\}(p)=\int_{0}^{\infty} t^{p-1} f(t) \mathrm{d} t \quad(p \in \mathbb{C}) .
$$

The domain of definition of the Mellin transform turns out to be an open strip of complex number $p=\sigma+i$ with $\sigma \in\langle a, b\rangle$. The largest open strip $\langle a, b\rangle$, where the integral converges, is called fundamental strip. 
Definition 2.8 (cf.[25], Inverse Mellin transform): Let $f \in L^{1}(0, \infty)$ with fundamental strip $\langle a, b\rangle$. If $\gamma$ is such that $a<\gamma<b$, and $\mathcal{M}\{f(t)\}(\gamma+\mathrm{i} t)$ integrable, then the equality

$$
\left(\mathcal{M}^{-1} M(p)\right)(x)=\frac{1}{2 \pi i} \int_{\gamma-\mathrm{i} \infty}^{\gamma+\mathrm{i} \infty} x^{-p} \mathcal{M}\{f(t)\}(p) \mathrm{d} p
$$

holds almost everywhere. Moreover, if $f$ is continuous, the equality holds everywhere on $(0, \infty)$.

Between the Laplace transform and Mellin transform the following equality is true.

$$
\mathcal{M}\{f(x)\}(p)=\frac{1}{\Gamma(1-p)} \mathcal{M}\{\mathcal{L}\{f(x)\}(s)\}(1-p) .
$$

Definition 2.9 (Fourier transform): Let $f \in L^{1}\left(\mathbb{R}^{n}\right)$, the Fourier transform of $f$ is defined by

$$
\mathfrak{F}\{f(x)\}(\xi)=\widehat{f}(\xi)=\frac{1}{(2 \pi)^{n / 2}} \int_{\mathbb{R}^{n}} \mathrm{e}^{-\mathrm{i}(\xi, x)} f(x) \mathrm{d} x,
$$

where $\xi=\left(\xi_{1}, \xi_{2}, \ldots, \xi_{n}\right), x=\left(x_{1}, x_{2}, \ldots x_{n}\right) \in \mathbb{R}^{n},(\xi, x)=\xi_{1} x_{1}+\cdots+\xi_{n} x_{n}$ and $d x=$ $d x_{1} d x_{2} \cdots d x_{n}$ and the inverse Fourier transform is given by

$$
\mathfrak{F}^{-1}\{\widehat{f}(\xi)\}(\xi)=\frac{1}{(2 \pi)^{n / 2}} \int_{\mathbb{R}^{n}} \mathrm{e}^{\mathrm{i}(\xi, x)} \widehat{f}(\xi) \mathrm{d} \xi .
$$

\section{Ultra-hyperbolic time-fractional diffusion-wave equation}

In this section we solve a Cauchy problem associated with the time-fractional diffusionwave equation, which results from replacing the Laplacian operator by ultra-hyperbolic operator.

Let us consider the following Cauchy problem :

$$
\begin{aligned}
D_{t}^{\alpha, r} u(x, t)-c^{2} \square u(x, t) & =0, \quad t>0 ; x \in D, \\
\left.I^{(2-\alpha)(1-r)} u(x, t)\right|_{t=0} & =f(x), \\
\left.\frac{\partial}{\partial t} I^{(2-\alpha)(1-r)} u(x, t)\right|_{t=0} & =g(x) .
\end{aligned}
$$

where $D_{t}^{\alpha, r}$ is the Hilfer derivative (2.11) of order $1<\alpha \leq 2$, and type $0 \leq r \leq 1$, the operator $\square$ is defined by

$$
\square=\left(\frac{\partial^{2}}{\partial x_{1}^{2}}+\cdots+\frac{\partial^{2}}{\partial x_{\mu}^{2}}-\frac{\partial^{2}}{\partial x_{\mu+1}^{2}}-\cdots-\frac{\partial^{2}}{\partial x_{\mu+\nu}^{2}}\right),
$$

$\mu+v=n$ is the dimension of the Euclidean space $\mathbb{R}^{n}, f, g \in L^{1}\left(\mathbb{R}^{n}\right)$ and $D$ is defined by

$$
\begin{aligned}
D= & \left\{\left(x_{1}, \ldots x_{\mu}, \ldots x_{\mu+\nu}\right) \in \mathbb{R}^{n}: \mu+v=n, x_{1}^{2}\right. \\
& \left.+x_{2}^{2}+\cdots+x_{\mu}^{2} \geq x_{\mu+1}^{2}+\cdots+x_{\mu+\nu}^{2}\right\} .
\end{aligned}
$$


To solve the Cauchy problem (3.1), first we apply Fourier transform with respect to the space variable

$$
D_{t}^{\alpha, r} \hat{u}(\xi, t)+c^{2}\left(\xi_{1}^{2}+\cdots+\xi_{\mu}^{2}-\xi_{\mu+1}^{2}-\cdots-\xi_{\mu+v}^{2}\right) \hat{u}(\xi, t)=0 .
$$

Now, applying the Laplace transform in the time variable to both sides of the above equation and putting

$$
\begin{aligned}
& \|\xi\|_{\mu, v}^{2}=\xi_{1}^{2}+\cdots+\xi_{\mu}^{2}-\xi_{\mu+1}^{2}-\cdots-\xi_{\mu+v}^{2}, \\
& s^{\alpha} \tilde{\hat{u}}(\xi, s)-s^{1-r(2-\alpha)} I^{(1-r)(2-\alpha)} \hat{u}(\xi, 0)-s^{-r(2-\alpha)} \frac{\partial}{\partial t} I^{(1-r)(2-\alpha)} \hat{u}(x, 0) \\
& \quad+c^{2}\|\xi\|_{\mu, \nu}^{2} \tilde{\hat{u}}(\xi, s)=0
\end{aligned}
$$

using the initial conditions

$$
\begin{aligned}
& s^{\alpha} \tilde{\hat{u}}(\xi, s)-s^{1-r(2-\alpha)} \hat{f}(\xi)-s^{-r(2-\alpha)} \hat{g}(x)+c^{2}\|\xi\|_{\mu, \nu}^{2} \tilde{\hat{u}}(\xi, s)=0, \\
& \tilde{\hat{u}}(\xi, s)=\frac{s^{1-r(2-\alpha)}}{s^{\alpha}+c^{2}\|\xi\|_{\mu, \nu}^{2}} \hat{f}(\xi)+\frac{s^{-r(2-\alpha)}}{s^{\alpha}+c^{2}\|\xi\|_{\mu, \nu}^{2}} \hat{g}(\xi) .
\end{aligned}
$$

Taking inverse Laplace transform is that

$$
\begin{aligned}
\hat{u}(\xi, t)= & t^{(1-r) \alpha+2 r-2} E_{\alpha,(1-r) \alpha+2 r-1}\left(-c^{2}\|\xi\|_{\mu, \nu}^{2} t^{\alpha}\right) \hat{f}(\xi) \\
& +t^{(1-r) \alpha+2 r-1} E_{\alpha,(1-r) \alpha+2 r}\left(-c^{2}\|\xi\|_{\mu, \nu}^{2} t^{\alpha}\right) \hat{g}(\xi) .
\end{aligned}
$$

Then, by inverse Fourier transform

$$
\begin{aligned}
u(x, t)= & \mathfrak{F}^{-1}\left\{t^{(1-r) \alpha+2 r-2} E_{\alpha,(1-r) \alpha+2 r-1}\left(-c^{2}\|\xi\|_{\mu, \nu}^{2} t^{\alpha}\right)\right\} * f(x) \\
& +\mathfrak{F}^{-1}\left\{t^{(1-r) \alpha+2 r-1} E_{\alpha,(1-r) \alpha+2 r}\left(-c^{2}\|\xi\|_{\mu, \nu}^{2} t^{\alpha}\right)\right\} * g(x) .
\end{aligned}
$$

To calculate $\mathfrak{F}^{-1}\left\{t^{(1-r) \alpha+2 r-2} E_{\alpha,(1-r) \alpha+2 r-1}\left(-c^{2}\|\xi\|_{\mu, \nu}^{2} t^{\alpha}\right)\right\} \quad$ y $\mathfrak{F}^{-1}\left\{t^{(1-r) \alpha+2 r-1}\right.$ $\left.E_{\alpha,(1-r) \alpha+2 r}\left(-c^{2}\|\xi\|_{\mu, v}^{2} t^{\alpha}\right)\right\}$ use the procedure followed by Yakubovich and Luchko, [16]. To do this, consider

$$
\mathcal{E}(t, \xi ; \alpha, \beta) \doteq t^{\beta-1} E_{\alpha, \beta}\left( \pm D\|\xi\|_{\mu, \nu}^{2} t^{\alpha}\right),
$$

where $D$ is a positive constant and $\|\xi\|_{\mu, \nu}^{2}$ is given by (3.5).

It is known that the Laplace transform of the function $\mathcal{E}(t, \xi ; \alpha, \beta)$ with respect the time variable $t$ is given by

$$
\mathcal{L}\{\mathcal{E}(t, \xi ; \alpha, \beta)\}(s)=\frac{s^{\alpha-\beta}}{s^{\alpha} \mp D\|\xi\|_{\mu, v}^{2}} .
$$

Now we calculate the Mellin transform of $\mathcal{E}(t, \xi ; \alpha, \beta)$ with respect to $t$ using the relation (2.19). Then

$$
\mathcal{M}\{\mathcal{E}(t, \xi ; \alpha, \beta)\}(p)=\frac{1}{\Gamma(1-p)} \int_{0}^{\infty} \frac{s^{\alpha-\beta-p}}{s^{\alpha} \mp D\|\xi\|_{\mu, \nu}^{2}} \mathrm{~d} s .
$$


Making the change of variables $s=\left(\mp D\|\xi\|_{\mu, \nu}^{2}\right)^{1 / 2} \theta$, result

$$
\mathcal{M}\{\mathcal{E}(t, \xi ; \alpha, \beta)\}(p)=\frac{\left[\mp D\|\xi\|_{\mu, \nu}^{2}\right]^{(1-\beta-p) / \alpha}}{\Gamma(1-p)} \int_{0}^{\infty} \frac{\theta^{\alpha-\beta-p}}{\theta^{\alpha}+1} \mathrm{~d} \theta .
$$

Taking $\theta=t^{1 / \alpha}$, we have by definition of Beta function,

$$
\int_{0}^{\infty} \frac{\theta^{\alpha-\beta-p}}{\theta^{\alpha}+1} \mathrm{~d} \theta=\frac{1}{\alpha} B\left(\frac{\beta+p-1}{\alpha}, \frac{1-\beta-p}{\alpha}+1\right) .
$$

Finally, from (3.14)-(3.16), we have that

$$
\mathcal{M}\{\mathcal{E}(t, \xi ; \alpha, \beta)\}(p)=\frac{\left[\mp D\|\xi\|_{\mu, v}^{2}\right]^{(1-\beta-p) / \alpha}}{\Gamma(1-p) \alpha} B\left(\frac{\beta+p-1}{\alpha}, \frac{1-\beta-p}{\alpha}+1\right) .
$$

\subsection{Inverse Fourier transform of a Mittag-Leffler-type function containing in its argument a positive-definite quadratic form}

Now let us calculate the inverse Fourier transform with respect to the variable $\xi$ of the $\mathcal{E}(t, \xi ; \alpha, \beta)$ function.

Given $\overline{\mathcal{E}}(t, x ; \alpha, \beta)=\mathfrak{F}^{-1}\{\mathcal{E}(t, \xi ; \alpha, \beta)\}(x)$, then, taking the Mellin transform both sides with respect to $t$ result

$$
\begin{aligned}
\mathcal{M}\{\overline{\mathcal{E}}(t, x ; \alpha, \beta)\}(p)= & \mathfrak{F}^{-1}\{\mathcal{M}\{\mathcal{E}(t, \xi ; \alpha, \beta)\}(p)\}(x) \\
= & \frac{(\mp D)^{(1-\beta-p) / \alpha}}{\alpha \Gamma(1-p)} \Gamma\left(\frac{\beta+p-1}{\alpha}\right) \Gamma\left(\frac{1-\beta-p}{\alpha}+1\right) \\
& \times \mathfrak{F}^{-1}\left\{\left(\|\xi\|_{\mu, \nu}^{2}\right)^{(1-\beta-p) / \alpha}\right\}(x) .
\end{aligned}
$$

To calculate $\mathfrak{F}^{-1}\left\{\left(\|\xi\|_{\mu, \nu}^{2}\right)^{(1-\beta-p) / \alpha}\right\}(x)$ we use a result due to Aguirre (cf.[26], f 2.3)

$$
\mathfrak{F}\left\{\left(\|x\|_{\mu, \nu}^{2}\right)^{\lambda}\right\}(\xi)=\frac{\mathrm{e}^{\nu \pi \mathrm{i} / 2} 2^{2 \lambda+n} \pi^{n / 2} \Gamma(\lambda+n / 2)}{\Gamma(-\lambda)}\left(\|\xi\|_{\mu, \nu}^{2}\right)^{-\lambda-n / 2} .
$$

This formula is valid if $x_{1}^{2}+x_{2}^{2}+\cdots+x_{\mu}^{2} \geq x_{\mu+1}^{2}+\cdots+x_{\mu+v}^{2}$ and $\xi_{1}^{2}+\xi_{2}^{2}+\cdots+$ $\xi_{\mu}^{2} \geq \xi_{\mu+1}^{2}+\cdots+\xi_{\mu+v}^{2}$.

Then, for $\lambda=(p+\beta-1) / \alpha-n / 2$, we have $\mathfrak{F}^{-1}\left\{\left(\|\xi\|_{\mu, \nu}^{2}\right)^{(1-\beta-p) / \alpha}\right\}(x)=\frac{\mathrm{i}^{v} \Gamma(n / 2-((p+\beta-1) / \alpha))}{2^{2((p+\beta-1) / \alpha)} \pi^{n / 2} \Gamma((p+\beta-1) / \alpha)}\left(\|x\|_{\mu, \nu}^{2}\right)^{(p+\beta-1) / \alpha-n / 2}$ then, returning to (3.18)

$$
\begin{aligned}
\mathcal{M}\{\overline{\mathcal{E}}(t, x ; \alpha, \beta)\}(p)= & \frac{(\mp D)^{(1-\beta-p) / \alpha}}{\alpha \Gamma(1-p)} \Gamma\left(\frac{1-\beta-p}{\alpha}+1\right) \\
& \times \frac{\mathrm{i}^{v} \Gamma(n / 2-((p+\beta-1) / \alpha))}{2^{2((p+\beta-1) / \alpha)} \pi^{n / 2}}\left(\|x\|_{\mu, v}^{2}\right)^{(p+\beta-1) / \alpha-n / 2} .
\end{aligned}
$$


Taking inverse Mellin transform results

$$
\begin{aligned}
\overline{\mathcal{E}}(t, x & ; \alpha, \beta)=\frac{\mathrm{i}^{v}\left(\|x\|_{\mu, \nu}^{2}\right)^{(\beta-1) / \alpha-n / 2}(\mp D)^{(1-\beta) / \alpha}}{\alpha 4^{(\beta-1) / \alpha} \pi^{n / 2}} \\
& \times \frac{1}{2 \pi i} \int_{p-\mathrm{i} \infty}^{p+\mathrm{i} \infty} \frac{\Gamma((1-\beta-p) / \alpha+1) \Gamma((1-\beta-p) / \alpha+n / 2)}{\Gamma(1-p)} \\
& \times\left[\left(\frac{\|x\|_{\mu, v}^{2}}{4(\mp D) t^{\alpha}}\right)^{1 / \alpha}\right] p \mathrm{~d} p=\frac{\mathrm{i}^{q}\left(\|x\|_{\mu, \nu}^{2}\right)^{(\beta-1) / \alpha-n / 2}(\mp D)^{(1-\beta) / \alpha}}{\alpha 4^{(\beta-1) / \alpha} \pi^{n / 2}} \\
& \times \frac{1}{2 \pi i} \int_{p-\mathrm{i} \infty}^{p+\mathrm{i} \infty} \frac{\Gamma((1-\beta-p) / \alpha+1) \Gamma((1-\beta-p) / \alpha+n / 2)}{\Gamma(1-p)} \\
& \times\left[\left(\frac{4(\mp D) t^{\alpha}}{\|x\|_{\mu, \nu}^{2}}\right)^{1 / \alpha}\right]^{-p} \mathrm{~d} p \\
= & \frac{\mathrm{i}^{v}\left(\|x\|_{\mu, \nu}^{2}\right)^{(\beta-1) / \alpha-n / 2}(\mp D)^{(1-\beta) / \alpha}}{\alpha 4^{(\beta-1) / \alpha} \pi^{n / 2}} \\
\times & \frac{1}{2 \pi i} \int_{p-\mathrm{i} \infty}^{p+\mathrm{i} \infty} \frac{\Gamma(1-((\beta-1) / \alpha)-(1 / \alpha) p) \Gamma(1-((\beta+\alpha-1) / \alpha-n / 2)-(1 / \alpha) p)}{\Gamma(1-p)} \\
\times & {\left[\left(\frac{4(\mp D) t^{\alpha}}{\|x\|_{\mu, v}^{2}}\right)^{1 / \alpha}\right]^{-p} \mathrm{~d} p . }
\end{aligned}
$$

From the definition of the H-function, for $m=0, n=2, p=2, q=1$, and $a_{1}=$ $(\beta-\alpha) / \alpha, A_{1}=1 / \alpha, a_{2}=(\beta-\alpha-1) / \alpha-n / 2, A_{2}=1 / \alpha, b_{1}=0, B_{1}=1$

$$
\left|\arg \left(\frac{4(\mp D) t^{\alpha}}{\|x\|_{\mu, \nu}^{2}}\right)^{1 / \alpha}\right|<\frac{C \pi}{2}
$$

with $C=(2+\alpha) / \alpha>0$, we have

$$
\begin{aligned}
\overline{\mathcal{E}}(t, x ; \alpha, \beta)= & \frac{i^{\nu}\left(\|x\|_{\mu, \nu}^{2}\right)^{(\beta-1) / \alpha-n / 2}(\mp D)^{(1-\beta) / \alpha}}{\alpha 4^{(\beta-1) / \alpha} \pi^{n / 2}} \\
& \times H_{2,1}^{0,2}\left(\left(\frac{4(\mp D) t^{\alpha}}{\|x\|_{\mu, \nu}^{2}}\right)^{1 / \alpha} \mid\left(\frac{\beta-1}{\alpha}, \frac{1}{\alpha}\right),\left(\frac{\beta+\alpha-1}{\alpha}-\frac{n}{2}, \frac{1}{\alpha}\right)\right) \\
= & \frac{i^{\nu} t^{\beta-1}}{\left(4 \pi(\mp D) t^{\alpha}\right)^{n / 2}}\left[\frac{\|x\|_{\mu, \nu}^{2}}{4(\mp D) t^{\alpha}}\right]^{(\beta-1) / \alpha-n / 2} \\
& \times \frac{1}{\alpha} H_{2,1}^{0,2}\left(\left(\frac{4(\mp D) t^{\alpha}}{\|x\|_{\mu, \nu}^{2}}\right)^{1 / \alpha} \mid\left(\frac{\beta-1}{\alpha}, \frac{1}{\alpha}\right),\left(\frac{\beta+\alpha-1}{\alpha}-\frac{n}{2}, \frac{1}{\alpha}\right)\right)
\end{aligned}
$$


from (2.5) and (2.6) result

$$
\begin{aligned}
\overline{\mathcal{E}}(t, x ; \alpha, \beta)= & \frac{\mathrm{i}^{v} t^{\beta-1}}{\left(4 \pi(\mp D) t^{\alpha}\right)^{n / 2}}\left[\frac{\|x\|_{\mu, \nu}^{2}}{4(\mp D) t^{\alpha}}\right]^{(\beta-1) / \alpha-n / 2} \\
& \times H_{1,2}^{2,0}\left(\frac{\|x\|_{\mu, \nu}^{2}}{4(\mp D) t^{\alpha}} \mid\left(1-\left(\frac{\beta-1}{\alpha}\right), 1\right),\left(\frac{n}{2}-\left(\frac{\beta-1}{\alpha}\right), 1\right)\right) .
\end{aligned}
$$

Now, using (2.7) we have

$$
\begin{aligned}
& \overline{\mathcal{E}}(t, x ; \alpha, \beta)=\frac{\mathrm{i}^{\nu} t^{\beta-1}}{\left(4 \pi(\mp D) t^{\alpha}\right)^{n / 2}} \\
& \quad \times H_{1,2}^{2,0}\left(\frac{\|x\|_{\mu, \nu}^{2}}{4(\mp D) t^{\alpha}} \mid\left(1+\left(\frac{\beta-1}{\alpha}-\frac{n}{2}\right) \alpha, \alpha\right)\right. \\
& \left.\left.\quad\left(\frac{\beta-1}{\alpha}\right)+\frac{\beta-1}{\alpha}-\frac{n}{2}, 1\right),\left(\frac{n}{2}-\left(\frac{\beta-1}{\alpha}\right)+\frac{\beta-1}{\alpha}-\frac{n}{2}, 1\right)\right),
\end{aligned}
$$

i.e.

$$
\begin{aligned}
\mathfrak{F}^{-1}\{\mathcal{E}(t, \xi ; \alpha, \beta)\}(x)= & \frac{\mathrm{i}^{v} t^{\beta-1}}{\left(4 \pi(\mp D) t^{\alpha}\right)^{n / 2}} \\
& \times H_{1,2}^{2,0}\left(\begin{array}{l|c}
\|x\|_{\mu, v}^{2} & \left(\beta-\frac{n \alpha}{2}, \alpha\right) \\
4(\mp D) t^{\alpha} & \left(1-\frac{n}{2}, 1\right),(0,1)
\end{array}\right) .
\end{aligned}
$$

Returning to (3.11) and using (3.26) for cases

$$
\beta=(1-r) \alpha+2 r-1
$$

and

$$
\beta=(1-r) \alpha+2 r
$$

we have that the solution of the Cauchy problem (3.1) is

$$
\begin{aligned}
u(x, t)= & \frac{\mathrm{i}^{v} t^{(1-r) \alpha+2 r-2}}{\left(4 \pi c^{2} t^{\alpha}\right)^{n / 2}} \\
& \times H_{1,2}^{2,0}\left(\frac{\|x\|_{\mu, v}^{2}}{4 c^{2} t^{\alpha}} \mid \begin{array}{c}
\left((1-r) \alpha+2 r-1-\frac{n \alpha}{2}, \alpha\right) \\
\left(1-\frac{n}{2}, 1\right),(0,1)
\end{array}\right) * f(x) \\
& +\frac{\mathrm{i}^{v} t^{(1-r) \alpha+2 r-1}}{\left(4 \pi c^{2} t^{\alpha}\right)^{n / 2}} \\
& \times H_{1,2}^{2,0}\left(\frac{\|x\|_{\mu, v}^{2}}{4 c^{2} t^{\alpha}} \mid \begin{array}{c}
\left((1-r) \alpha+2 r-\frac{n \alpha}{2}, \alpha\right) \\
\left(1-\frac{n}{2}, 1\right),(0,1)
\end{array}\right) * g(x)
\end{aligned}
$$

provided that $x_{1}^{2}+x_{2}^{2}+\cdots+x_{\mu}^{2} \geq x_{\mu+1}^{2}+\cdots+x_{\mu+\nu}^{2}$. 


\subsection{Particular cases}

(1) Note that if $r=1$, the Cauchy problem (3.1) it is expressed in terms of the Caputo fractional derivative

$$
\begin{aligned}
& { }^{c} D_{t}^{\alpha} u(x, t)-c^{2} \square u(x, t)=0 ; \\
& u(x, 0)=f(x), \\
& \left.\frac{\partial}{\partial t} u(x, t)\right|_{t=0}=g(x)
\end{aligned}
$$

and then, from (3.27) the solution is

$$
\begin{aligned}
u(x, t)= & \frac{\mathrm{i}^{v}}{\left(4 \pi c^{2} t^{\alpha}\right)^{n / 2}} \\
& \times H_{1,2}^{2,0}\left(\frac{\|x\|_{\mu, \nu}^{2}}{4 c^{2} t^{\alpha}} \mid \begin{array}{c}
\left(1-\frac{n \alpha}{2}, \alpha\right) \\
\left(1-\frac{n}{2}, 1\right),(0,1)
\end{array}\right) * f(x) \\
& +\frac{\mathrm{i}^{v} t}{\left(4 \pi c^{2} t^{\alpha}\right)^{n / 2}} \\
& \times H_{1,2}^{2,0}\left(\frac{\|x\|_{\mu, \nu}^{2}}{4 c^{2} t^{\alpha}} \mid \begin{array}{c}
\left(2-\frac{n \alpha}{2}, \alpha\right) \\
\left(1-\frac{n}{2}, 1\right),(0,1)
\end{array}\right) * g(x) .
\end{aligned}
$$

For $x_{1}^{2}+x_{2}^{2}+\cdots+x_{\mu}^{2} \geq x_{\mu+1}^{2}+\cdots+x_{\mu+v}^{2}$.

(2) If $r=0$, the Cauchy problem (3.1) is expressed in terms of the Riemann-Liouville fractional derivative

$$
\begin{aligned}
& { }^{R-L} D_{t}^{\alpha} u(x, t)-c^{2} \square u(x, t)=0 ; \\
& I^{2-\alpha} u(x, 0)=f(x), \\
& \left.\frac{\partial}{\partial t} I^{2-\alpha} u(x, t)\right|_{t=0}=g(x)
\end{aligned}
$$

and then, from (3.27) the solution is

$$
\begin{aligned}
u(x, t)= & \frac{\mathrm{i}^{v} t^{\alpha-2}}{\left(4 \pi c^{2} t^{\alpha}\right)^{n / 2}} \\
& \times H_{1,2}^{2,0}\left(\frac{\|x\|_{\mu, \nu}^{2}}{4 c^{2} t^{\alpha}} \mid\left(\alpha-1-\frac{n \alpha}{2}, \alpha\right)\right) * f(x) \\
& \left.\left.+\frac{\mathrm{i}^{v} t^{\alpha-1}}{\left(4 \pi c^{2} t^{\alpha}\right)^{n / 2}}, 1\right),(0,1)\right) \\
& \times H_{1,2}^{2,0}\left(\frac{\|x\|_{\mu, \nu}^{2}}{4 c^{2} t^{\alpha}} \mid \begin{array}{c}
\left(\alpha-\frac{n \alpha}{2}, \alpha\right) \\
\left(1-\frac{n}{2}, 1\right),(0,1)
\end{array}\right) * g(x) .
\end{aligned}
$$

Provided that $x_{1}^{2}+x_{2}^{2}+\cdots+x_{\mu}^{2} \geq x_{\mu+1}^{2}+\cdots+x_{\mu+v}^{2}$. 
(3) Now, if $\alpha \downarrow 1$ and $r=1$, the problem (3.1) is reduced to the Cauchy problem (3.1)-(3.2) of Nonlaopon and Kananthai,[14] and the solution is given by

$$
\begin{aligned}
u(x, t)= & \frac{\mathrm{i}^{v}}{\left(4 \pi c^{2} t\right)^{n / 2}} \\
& \times H_{1,2}^{2,0}\left(\begin{array}{l|c}
\frac{\|x\|_{\mu, \nu}^{2}}{4 c^{2} t} & \left(1-\frac{n}{2}, 1\right) \\
\left(1-\frac{n}{2}, 1\right),(0,1)
\end{array}\right) * f(x)
\end{aligned}
$$

but, as

$$
\begin{aligned}
H_{1,2}^{2,0}\left(\frac{\|x\|_{\mu, v}^{2}}{4 c^{2} t} \mid \begin{array}{c}
\left(1-\frac{n}{2}, 1\right) \\
\left(1-\frac{n}{2}, 1\right),(0,1)
\end{array}\right) & =\sum_{p=0}^{\infty} \operatorname{Res}\left(\Gamma(-p)\left[\frac{\|x\|_{\mu, v}^{2}}{4 c^{2} t}\right]^{p}\right) \\
& =\sum_{p=0}^{\infty} \frac{(-1)^{p}}{p !}\left[\frac{\|x\|_{\mu, v}^{2}}{4 c^{2} t}\right]^{p} \\
& =\exp \left(-\frac{\|x\|_{\mu, v}^{2}}{4 c^{2} t}\right)
\end{aligned}
$$

finally results that (3.32) coincides with (3.3) of Nonlaopon and Kananthai,[14] provided that $x_{1}^{2}+x_{2}^{2}+\cdots+x_{\mu}^{2} \geq x_{\mu+1}^{2}+\cdots+x_{\mu+v}^{2}$.

(4) On the other hand, if $\alpha=2$ and $r$ take any value, the problem (3.1) is reduced to the Cauchy problem (8)-(9) when $k=1$ of Sansanit and Kananthai [15] and the explicit solution is given by

$$
\begin{aligned}
u(x, t)= & \frac{\mathrm{i}^{\nu}}{\left(4 \pi c^{2} t^{2}\right)^{n / 2}} \\
& \times H_{1,2}^{2,0}\left(\frac{\|x\|_{\mu, \nu}^{2}}{4 c^{2} t^{2}} \mid \begin{array}{c}
(1-n, 2) \\
\left(1-\frac{n}{2}, 1\right),(0,1)
\end{array}\right) * f(x) \\
& +\frac{\mathrm{i}^{v} t}{\left(4 \pi c^{2} t^{2}\right)^{n / 2}} \\
& \times H_{1,2}^{2,0}\left(\frac{\|x\|_{\mu, \nu}^{2}}{4 c^{2} t^{2}} \mid\left(1-\frac{n}{2}, 1\right),(0,1)\right) * g(x) .
\end{aligned}
$$

For $x_{1}^{2}+x_{2}^{2}+\cdots+x_{\mu}^{2} \geq x_{\mu+1}^{2}+\cdots+x_{\mu+v}^{2}$.

Remark 3: An important question about this solution is that it was not given explicitly in [15].

\section{Disclosure statement}

No potential conflict of interest was reported by the author. 


\section{References}

[1] Fujita Y. Cauchy problems of fractional order and stable processes. Japan J Appl Math. 1990;7(3):459-476.

[2] Nigmatullin RR. The realiztion of the generalized transfer equation in a medium with fractal geometry. Phys Status Solidi B. 1986;133(1):425-430.

[3] Wyss W. The fractional diffusion equation. J Math Phys. 1986;27:2782-2785.

[4] Schneider WR, Wyss W. Fractional diffusion and wave equations. J Math Phys. 1989;30: 134-144.

[5] Mainardi F. The fundamental solutions for the fractional diffusion-wave equation. Appl Math Lett. 1996;9(6):23-28.

[6] Hilfer R. Fractional diffusion based on Riemann-Liouville fractional derivatives. J Phys Chem B. 2000;104(16):3914-3917.

[7] Huang F, Liu F. The time fractional diffusion and wave equations in an n-dimensional half space with mixed boundary conditions. Pacific J App Math. 2008;1(4):67-77.

[8] Sandev T, Metzler R, Tomovski Ž. Fractional diffusion equation with a generalized Riemann-Liouville time fractional derivative. J Phys A: Math Theor. 2011;44(25):255203.

[9] Tomovski Ž, Sandev T, Metzler R, Dubbeldam R. Generalized space-time fractional diffusion equation with composite fractional time derivative. Phys A: Statist Mech Appl. 2012;391(8):2527-2542.

[10] Gorenflo R, Mainardi F. Approximation of Lévy-Feller diffusion by random walk. J Anal Appl. 1999;18(2):231-246.

[11] Yu R, Zhang H. New function of Mittag-Leffler type and its application in the fractional diffusion-wave equation. Chaos Solitios Fractals. 2006;30(4):946-955.

[12] Gorenflo R, Luchko Y, Yamamoto M. Time-Fractional diffusion equation in the fractional Sobolev Spaces. Fract Calculus Appl Anal. 2015;18:799-820.

[13] Kochubei AN. Fractional order difussion. J Diff Eqs. 1990;26(4):485-492.

[14] Nonlaopon K, Kananthai A. On the Ultra-hyperbolic heat kernel. Int J Appl Math. 2003;13(2):215-225.

[15] Sansanit W, Kananthai A. On the Ultra-hyperbolic wave operator. Int J P Appl Math. 2009;52(1):117-126.

[16] Yakubovich SB, Luchko Y, The hypergeometric approach to integral transforms and convolutions, Mathematics and its applications, Vol. 287. Dordrecht: Kluwer Academic Publishers; 1994.

[17] Rodrigues MM, Vieira N, Yakubovich S. Operational calculus for Bessel's fractional equation. In: Almeida A, Castro L, Speck FO, editors. Advances in harmonic analysis and operator theory. Vol 229, Operator theory: advances and applications. Basel: Springer AG; 2013. p. 357-370.

[18] Yakubovich S, Rodrigues MM. Fundamental solutions of the fractional two-parameter telegraph equation. Integral Transforms Spec Funct. 2012;23(7):509-519.

[19] Dalla Riva M, Yakubovich S. On a Riemann-Liouville fractional analog of the Laplace operator with positive energy. Integral Transforms Spec Funct. 2012;23(4):277-295.

[20] Yakubovich S. Eigenfunctions and fundamental solutions of the fractional two-parameter Laplacian. Int J Math Math Sci. 2010;2010:18 p.

[21] Haubold HJ, Mathai AM, Saxena RK. Mittag-Leffler functions and their applications. J App Math. 2011;2011:51 p.

[22] Fox C. The G and $\mathrm{H}$ functions and as symmetrical Fourier kernels. Trans Amer Math Soc. 1961;98:395-429.

[23] Mathai AM, Saxena RK, Houbold HJ. The H-function: theory and applications. New York: Springer; 2010

[24] Tomovski Ž. Generalized Cauchy type problems for nonlinear fractional differential equations with composite fractional derivative operator. Nonlinear Anal: Theory, Methods Appl. 2012;75(7):3364-3384.

[25] Flajolet P, Gourdon X, Dumas P. Mellin transforms and asymptotics: harmonic sums. Theoret Comput Sci. 1995;144(1):3-58.

[26] Aguirre MA. A generalization of the fourier transform of Ultrahyperbolic operator. Thai J Math. 2013;11(3):499-518. 